\title{
ON CLAPEYRON'S EQUATION
}

\author{
BY PACL SACREI.
}

Consider at the temperature $T$ and under the pressure $\Pi$ a system whose energy, entropy and volume we shall denote by $\mathrm{E}, \mathrm{H}$ and $\mathrm{V}$. For a reversible change which leaves the mass of the system unchanged we have the well-known equation

$$
d \mathrm{E}=\mathrm{T} d \mathrm{H}-\mathrm{IH} d \mathrm{~V} \text {. }
$$

The total thermodynamic potential $\Phi$ is defined by the equation

$$
\Phi=\mathrm{E} \ldots \mathrm{TH}+\mathrm{TH} \mathrm{V} \text {. }
$$

Making use of equation I we obtain

$$
d \Phi=-\mathrm{H} d \mathrm{~T}+\mathrm{V} d \Pi \text {. }
$$

Suppose that the system under consideration is a univariant systen. In that case $I I$ and $T$ are no longer independent variables; one is a function of the other, so that we may write

$$
\Pi=f(T) \text {. }
$$

Equation I shows that if we assume $\mathrm{E}$ to be a function of $\mathrm{H}$ and $V, \Pi$ and $T$ are also functions of $\mathrm{H}$ and $\mathrm{V}$ and are given by the equations

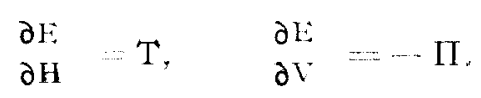

If equation 4 is to hold, the Jacobian of 11 and $T$ with respect to $\mathrm{H}$ and $V$ must vanish. That is to say, equation 4 is equivalent to the equation

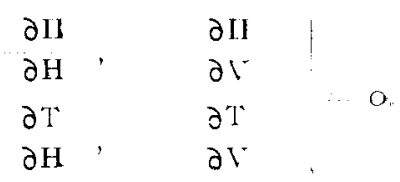


For the system under consideration, the thermodynamic potential $\Phi$ is also a function of $\Pi$ or of $T$. To show this it will be sufficient to prove that

$\begin{array}{ll}\partial \Phi & \partial \Phi \\ \partial H & \partial V \\ \partial T & \partial T \\ \partial H & \partial V\end{array} \mid=0$.

If we make use of equations 2 and 5 , this determinant takes the form

$$
\begin{aligned}
& -H \frac{\partial T}{\partial H}+V-\frac{\partial I I}{\partial H}, \quad-H \frac{\partial T}{\partial V}-V^{-} \frac{\partial \Pi}{\partial V} \\
& \partial \mathrm{T} \\
& \partial \mathrm{T} \\
& \partial \mathrm{H} \\
& \partial V
\end{aligned}
$$

This may easily be reduced to the form

$$
\begin{array}{lll}
\partial H & \frac{\partial \Pi}{\partial H} \\
\frac{\partial T}{\partial} & \partial T \\
\partial H & \partial V
\end{array},
$$

which, by equation 6 , is equal to zero. We have thus shown that $\Phi$ is a function of $T$.

Equation 3 may be written in the form

$$
\begin{aligned}
& d \Phi=\left[\begin{array}{ll}
--\mathrm{H}+\mathrm{V} & \partial \Pi \mathrm{T}^{-}
\end{array}\right] d \mathrm{~T} \\
& =\left[-\mathrm{H}+\mathrm{V} f^{\prime}(\mathrm{T})\right] d \mathrm{~T} \text {. }
\end{aligned}
$$

in which $f^{\prime}(\mathrm{T})$ denotes the derivative of the function which appears in equation 4. As $\Phi$ is a function of $T$, it follows that

$$
-\mathrm{H}+\mathrm{V} f^{\prime}
$$

must be a function of $T$. To express this algebraically, it is sufficient to write

$$
\begin{aligned}
& \frac{\partial}{\partial \mathrm{H}}\left[-\mathrm{H}+\mathrm{V} f^{\prime}(\mathrm{T})\right], \quad \frac{\partial}{\partial \mathrm{V}^{\prime}}\left[-\mathrm{H}-\mathrm{V} f^{\prime}(\mathrm{T})\right] \\
& \partial \mathrm{T} \\
& \partial \mathrm{T} \\
& \partial v
\end{aligned}
$$


On performing the indicated differentiations we get

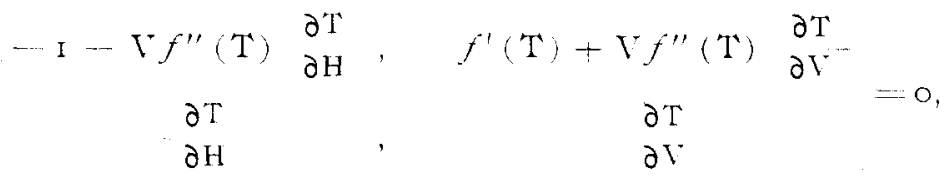

which reduces to

$$
\begin{array}{cc}
-\mathrm{I} & f^{\prime}(\mathrm{T}) \\
\partial \mathrm{T} & \partial \mathrm{T} \\
\partial \mathrm{H} & \partial \mathrm{T}
\end{array}
$$

If we expand this determinant we find that

$$
\begin{aligned}
f^{\prime}(\mathrm{T}) & =-\frac{\left(\begin{array}{l}
\partial \mathrm{T} \\
\partial \mathrm{V}
\end{array}\right)_{\mathrm{IH}}}{\left(\begin{array}{l}
\partial \mathrm{T} \\
\partial \mathrm{H}
\end{array}\right)_{\mathrm{V}}} \\
& =-\left(\begin{array}{l}
\partial \mathrm{T} \\
\partial \mathrm{V}
\end{array}\right)_{\mathrm{H}} \cdot\left(\begin{array}{c}
\partial \mathrm{H} \\
\partial \mathrm{T}
\end{array}\right)_{\mathrm{V}} \cdot
\end{aligned}
$$

If. finally, we make use of the well-known relation

$$
\left(\begin{array}{l}
\partial Y \\
\partial H
\end{array}\right)_{T} \cdot\left(\begin{array}{l}
\partial H \\
\partial T
\end{array}\right)_{V} \cdot\left(\begin{array}{l}
\partial T \\
\partial V
\end{array}\right)_{H}=-I,
$$

equation 9 takes the form of Clapeyron's equation,

$$
\frac{d \Pi}{d T}=\left(\begin{array}{l}
\partial \mathrm{H} \\
\partial \mathrm{V}
\end{array}\right)_{\mathrm{r}} .
$$

Sta bork, Februmy $27,190 \%$ 